\title{
A FIRST ANALYSIS OF STABILITY WHEN A CONSTRAINT ON THE SYSTEM STATE IS ENCOUNTERED
}

\author{
Ian Dobson \\ Department of Electrical and Computer Engineering \\ University of Wisconsin, Madison, WI, 53706 USA \\ e-mail: dobson@engr.wisc.edu
}

Abstract: When a slowly varying stable equilibrium of a vector field encounters a constraint, the dimension of the state space is reduced by one and the equilibrium instantaneously changes its degree of stability. We try to determine this change in stability as a function of the unconstrained system and the constraint. An application to improving the voltage stability of an electric power system near voltage collapse by selectively locking tapchanging transformers is briefly discussed.

\section{Introduction}

Many engineering systems are modelled by the vector field or differential equations $\dot{\mathbf{x}}=f(\mathbf{x})$ with state vector $\mathbf{x} \in \mathbf{R}^{n}$. The system is operated at a stable equilibrium $\mathbf{x}_{0}$ so that the system state $\mathbf{x}=\mathbf{x}_{0}$. If system parameters vary slowly, then $\mathbf{x}_{0}$ will vary slowly and the system state $\mathbf{x}$ will track $\mathbf{x}_{\mathbf{0}}$. However this model is idealised; in a practical system it is unusual for the state $\mathbf{x}$ to range over all of $\mathbf{R}^{n}$ because of limits or constraints on $\mathbf{x}$. For example, if the first component $x_{1}$ of $\mathbf{x}$ has a limit $x_{1 l i m}$, then, as parameters vary, $x_{1}$ may reach and stay at $x_{1 \text { lim }}$. The equations then change from $\dot{\mathbf{x}}=f(\mathbf{x})$

$$
\begin{aligned}
\text { or } & \dot{x}_{k}=f_{k}\left(x_{1}, x_{2}, \ldots, x_{n}\right), \quad k=1,2, \ldots, n \\
\text { to } & \dot{x}_{k}=f_{k}\left(x_{1 l i m}, x_{2}, \ldots, x_{n}\right), \quad k=2, \ldots, n .
\end{aligned}
$$

$x_{1}$ reaching its limit alters the vector field and reduces the state vector by one dimension to $\mathbf{y}=\left(x_{2}, x_{3}, \ldots, x_{n}\right)$. In geometric terms the state space is reduced to the hyperplane $C$ given by $x_{1}=x_{1 \max }$ and the vector field $f^{c}=\left(f_{2}, f_{3}, \ldots, f_{n}\right)$ on $C$ is the projection of the vector field $f$ onto $C$. Thus both the state space and the vector field change when a constraint or limit is encountered. In particular, the degree of stability of the system changes instantaneously when the constraint is encountered. In extreme cases, the system may become instantaneously unstable when the constraint is encountered; this is an interesting mechanism for system instability.

We investigate the effect of encountering a constraint and particularly its effect on system stability. In some systems, the constraints are not all preset and some may be applied at will so that the system may be controlled by selectively applying constraints. The same analysis applies to these constraints and determines whether it is advantageous for system stability to apply a given constraint (see the application in section 5).

We formulate the problem of a nonlinear vector field encountering a linear constraint and note that the stability of the constrained system is usually determined by the stability of the constrained linearization of the system. Therefore we study the stability of a linear system encountering a linear constraint and give conditions on the constraint guaranteeing stability or a certain change in the degree of stability when the constraint is encountered. Of course, the stability of a system encountering a constraint may be found simply by determining the constrained system and calculating the eigenvalues of its Jacobian at the operating equilibrium. However in this procedure, the relationship between the constraint, the stability of the system and the stability of the constrained system is not apparent and no analytic study is practical except on a numerical, case by case basis. Our objective is to study the stability of the constrained system directly from the stability of the system and the constraint without explicitly determining the constrained system. This allows analytic study of the process of encountering a constraint.

\section{A nonlinear system encountering a constraint}

We consider applying linear constraints to the nonlinear system

$$
\dot{\mathbf{x}}=f_{\mu}(\mathbf{x}), \quad \mathbf{x} \in \mathbf{R}^{n} .
$$

$\mu$ is a parameter which we often omit from the notation for $f$. The system state $\mathbf{x}$ is assumed to track a stable equilibrium $\mathbf{x}_{0}$ which varies as $\mu$ varies quasistatically. $\mathbf{A}$ linear constraint is specified by a hyperplane $C$ in $R^{n}$. We generally assume that the constraint is satisfied by the current system state; that is, the hyperplane $C$ passes through $\mathbf{x}_{0}$. This assumption holds when the constraint is caused by a state variable encountering a limit or being frozen at its current value in an attempt to control the system.

If the unit normal to $C$ is the row vector $\mathbf{c}$ then $C$ is the set of $\mathbf{x} \in \mathbf{R}^{n}$ with $\mathbf{c}\left(\mathbf{x}-\mathbf{x}_{0}\right)=0$. Applying a linear constraint to the system has two effects: the system state is constrained to lie in $C$ and the vector field $f$ on $\mathbf{R}^{n}$ is 
projected onto $C$ to form the constrained vector field $f^{c}$. Note that $C$ is invariant under $f^{c}$. If we write $\pi_{c}$ for the projection onto a hyperplane through the origin parallel to $C$ and $\left.f\right|_{C}$ for the restriction of $f$ to $C$, then the constrained vector field is given by

$$
f^{c}(\mathbf{y})=\left.\pi_{c} f\right|_{C}(\mathbf{y}), \quad \mathbf{y} \in C
$$

$f^{c}$ has an equilibrium at $\mathbf{x}_{0}$ and at any $\mathbf{x} \in C$ such that $f(\mathbf{x})$ is normal to $C$.

The stability of the equilibrium $\mathbf{x}_{0}$ of $f^{c}$ is determined (except in marginal cases) by the stability of the linearization $D f^{c}\left(\mathbf{x}_{0}\right)$ and $D f^{c}\left(\mathbf{x}_{0}\right)=\left.\pi_{c} D f\left(\mathbf{x}_{0}\right)\right|_{C}$. Thus the effect of the constraint on the stability of $x_{0}$ may be reduced to the linear case studied in section 3 .

\section{A linear system encountering a constraint}

We study the linear case $f(x)=A x$ where $A$ is an $n \times n$ matrix. $A$ has an equilibrium at the origin and we assume that the constraint hyperplane $C$ contains the origin. The constrained vector field $f^{c}$ on $C$ is also linear and has matrix $A^{c}$ which we calculate as follows: Let $\left\{q_{1}, \ldots, q_{n-1}\right\}$ be a set of orthonormal vectors in $\mathbf{R}^{n}$ which are a basis for $C$. With respect to this basis, $\left.f\right|_{C}$ has matrix $A Q$, where the $r$ th column of the $n \times(n-1)$ matrix $\mathrm{Q}$ is $q_{r}, r=1, \ldots, n-1$. Also $\pi_{c}$ has matrix $Q^{T}$ since $\left\{q_{1}, \ldots, q_{n-1}\right\}$ are orthonormal. The matrix $A^{c}$ may now be calculated from (2):

$$
A^{c}=Q^{T} A Q
$$

The most basic question concerns stability when the constraint is encountered. For example, consider the stable system $A=\left(\begin{array}{cc}-1 & 5 \\ 0 & -2\end{array}\right)$ encountering the constraint $c=\frac{1}{\sqrt{2}}(1,-1)$. Then

$$
A^{c}=\frac{1}{\sqrt{2}}(1,1)\left(\begin{array}{cc}
-1 & 5 \\
0 & -2
\end{array}\right)\left(\begin{array}{l}
1 \\
1
\end{array}\right) \frac{1}{\sqrt{2}}=1
$$

which is unstable. Thus it is of interest to find conditions under which a stable linear system $A$ remains stable or is destabilized when a linear constraint is encountered.

The main results of the paper are contained in the series of lemmas in section 4 . Here we discuss the interpretation and use of the lemmas. Lemma 2 proves that $A^{c}$ is asymptotically stable for all constraints $\mathrm{c}$ if $A$ is negative definite and that if $A$ is not negative definite then there is a constraint $\mathrm{c}$ for which $A^{c}$ is not asymptotically stable. Lemmas 3,4 and 5 give an expression $\gamma(c)$ to calculate whether encountering a given constraint c improves or lessens stability. Suppose that $A$ is asymptotically stable with a real eigenvalue $\lambda$ which is closest to the imaginary axis and that we have calculated the right and left eigenvectors $\mathbf{v}$ and $\mathbf{w}$ corresponding to $\lambda$. (Recall that a left eigenvector of $A$ is a row vector satisfying $\mathbf{w} A=\lambda \mathbf{w}$.) We calculate the quantity

$$
\gamma(\mathbf{c})=(\mathbf{c v})\left(\mathbf{w c}^{T}\right) / \mathbf{w v}
$$

If $\gamma(\mathbf{c})$ is negative then $A^{c}$ has an eigenvalue with real part greater than $\lambda$ and hence $A^{c}$ is less stable than $A$.
If $\gamma(\mathbf{c})$ is positive then either no eigenvalues or a nonzero even number of eigenvalues of $A^{c}$ have real parts greater than $\lambda$. In the first case (no eigenvalues with real parts greater than $\lambda$ ) $A^{c}$ is more stable than $A$. In the second case (a nonzero even number of eigenvalues with real parts greater than $\lambda), A^{c}$ has become less stable than $A$ by movement of conjugate pairs of eigenvalues in the complex plane. Thus if $\gamma(\mathbf{c})$ is positive then $A^{c}$ could be more or less stable than $A$ but the manner in which $A^{c}$ can be less stable than $A$ is restricted.

For linear systems with symmetric $A$, the left eigenvector $\mathbf{w}$ is the transpose of the right eigenvector $\mathbf{v}$ and $\gamma(\mathbf{c})$ is always positive. Moreover, such a system can only have real eigenvalues. It follows from Lemma 5 that $A^{\mathrm{c}}$ is always more stable than $A$. This result generalizes to nonlinear systems on $\mathbf{R}^{n}$ with symmetric Jacobians; that is, gradient vector fields on $\mathbf{R}^{n}$.

The calculation of $\gamma(\mathbf{c})$ in (4) has a simple geometric interpretation: First choose the directions of $\mathbf{v}$ and $\mathbf{w}^{T}$ so that the angle between them is acute (i.e. wv $>0$ ). $\mathbf{c v}$ is the projection of $\mathbf{v}$ on $\mathbf{c}$ and is positive when $\mathbf{v}$ is on one side of $C$ and negative when $\mathbf{v}$ is on the other side of $C$. $\mathbf{w c}^{T}=\mathbf{c w}^{T}$ is the projection of $\mathbf{w}^{T}$ on $\mathbf{c}$ and is similarly positive or negative depending on which side of $C \mathbf{w}^{T}$ is. If $\mathbf{v}$ and $\mathbf{w}^{T}$ are on opposite sides of $C$ then $\mathbf{c v}$ and $\mathbf{w c}^{T}$ have opposite signs, $\gamma(\mathbf{c})$ is negative and applying the constraint lessens stability. If $\mathbf{v}$ and $\mathbf{w}^{T}$ are on the same side of $C$ then $\mathbf{c v}$ and $\mathbf{w c}^{T}$ have the same sign, $\gamma(\mathbf{c})$ is positive and applying the constraint might improve stability. Thus $\gamma(\mathbf{c})$ describes some of the stability changes due to the constraint $c$ and has a clear and geometric dependence on $\mathbf{v}, \mathbf{w}$ and $\mathbf{c}$. If there are many constraints to encounter or apply, then $\mathbf{v}$ and $\mathbf{w}$ need only be calculated once and the desirability or otherwise of the constraints may be tested according to the values of $\gamma$ on the constraints.

We have described theory for a constraint hyperplane $C$ passing through the equilibrium at the origin of a linear system $\dot{\mathbf{x}}=f(\mathbf{x})=A \mathbf{x}$. This theory extends easily to constraint hyperplanes $C$ that do not pass through the origin if we continue to assume that $f$ is linear. The constraint hyperplane $C$ is now given by $\mathbf{c x}=k$ for some nonzero constant $k$ and the constrained vector field is $f^{c}(\mathbf{y})=Q^{T} A\left(Q \mathbf{y}+k \mathbf{c}^{T}\right)$ which is no longer linear, but affine. (Note that if $Q^{T} A Q$ is invertible then $f^{c}$ has a unique equilibrium at $-k\left[Q^{T} A Q\right]^{-1} Q^{T} A \mathbf{c}^{T}$.) However the stability of $f^{c}$ is identical to the stability of $Q^{T} . A Q$ and so the results above extend immediately to this case. That is, when studying the stability of a linear system with a linear constraint, the constraint may be assumed to pass through the origin without loss of generality.

\section{Main Results}

Regard the hyperplane $C$ as a copy of $\mathrm{R}^{n-1}$ and write $i$ for the injection $C \rightarrow \mathbf{R}^{n}$ and $\pi_{c}$ for the projection $\mathbf{R}^{n} \rightarrow C$ along $\mathbf{c}$. Then $A^{\mathrm{c}}=\pi_{c} A i$. 
Lemma 1 (real eigenstructure of $A^{c}$ ) Let $|\mathbf{x}|=1$ and $|\mathbf{c}|=1$. Then $\mathbf{x} \in i(C) \subset \mathbf{R}^{n}$ and $\pi_{c} \mathbf{x}$ is a right eigenvector of $A^{c}$ with real eigenvalue $\lambda \Longleftrightarrow \mathbf{x}^{T} A \mathbf{x}=\lambda$ and

$$
\mathbf{c}= \begin{cases}(A \mathbf{x}-\lambda \mathbf{x})^{T} /|A \mathbf{x}-\lambda \mathbf{x}| & \text { if } A \mathbf{x}-\lambda \mathbf{x} \neq 0 \\ \text { any unit vector } \mathbf{c} \text { with } \mathbf{c x}=0 & \text { if } A \mathbf{x}-\lambda \mathbf{x}=0\end{cases}
$$

Proof : $\mathbf{x} \in i(C)$ implies that $\mathbf{c x}=0$. If $\pi_{c} \mathbf{x}$ is a right eigenvector of $A^{c}$ with eigenvalue $\lambda$ then

$$
\begin{array}{r}
0=i\left(A^{c} \pi_{c} \mathbf{x}-\lambda \pi_{c} \mathbf{x}\right)=i \pi_{c}(A \mathbf{x}-\lambda \mathbf{x})=\left(I-\mathbf{c}^{T} \mathbf{c}\right)(A \mathbf{x}-\lambda \mathbf{x}) \\
\text { and } \quad \mathbf{c}^{T} \mathbf{c} A \mathbf{x}=A \mathbf{x}-\lambda \mathbf{x}
\end{array}
$$

Multiplying (5) on the left by $\mathbf{x}^{T}$ gives $\mathbf{x}^{T} A \mathbf{x}=\lambda \mathbf{x}^{T} \mathbf{x}=$ $\lambda$. If $A \mathbf{x}-\lambda \mathbf{x}=0$ then we are done. If $A \mathbf{x}-\lambda \mathbf{x} \neq 0$ then (5) implies that $\mathbf{c} A \mathbf{x} \neq 0$ and $\mathbf{c}^{T}=(A \mathbf{x}-\lambda \mathbf{x}) / \mathbf{c} A \mathbf{x}$. It follows that $\mathbf{c}=(A \mathbf{x}-\lambda \mathbf{x})^{T} /|A \mathbf{x}-\lambda \mathbf{x}|$ since $|\mathbf{c}|=1$.

If $\mathbf{c x}=0$ and $A \mathbf{x}-\lambda \mathbf{x}=0$ then $\mathbf{x} \in i(C)$ and $\pi_{c} \mathbf{x} \in$ $C$ and hence $A^{c} \pi_{c} \mathbf{x}=\pi_{c} A \mathbf{x}=\pi_{c} \lambda \mathbf{x}=\lambda \pi_{c} \mathbf{x}$. If $\mathbf{x}^{T} A \mathbf{x}=$ $\lambda$ and $A \mathbf{x}-\lambda \mathbf{x} \neq 0$ and $\mathbf{c}=(A \mathbf{x}-\lambda \mathbf{x})^{T} /|A \mathbf{x}-\lambda \mathbf{x}|$ then $\mathbf{c x}=\left(\mathbf{x}^{T} A \mathbf{x}-\lambda\right) /|A \mathbf{x}-\lambda \mathbf{x}|=0$ so $\mathbf{x} \in i(C)$ and $\pi_{c} \mathbf{x} \in C$ and $A^{c} \pi_{c} \mathbf{x}-\lambda \pi_{c} \mathbf{x}=\pi_{c}(A \mathbf{x}-\lambda \mathbf{x})=\pi_{c}\left(I-\mathbf{c}^{T} \mathbf{c}\right)(A \mathbf{x}-\lambda \mathbf{x})$ which vanishes since $\mathbf{c}=(A \mathbf{x}-\lambda \mathbf{x})^{T} /|A \mathbf{x}-\lambda \mathbf{x}|$.

Lemma 2 (stability for all constraints) Let $k \in \mathbf{R}$. Then $A^{c}<k$ for all constraints $\mathrm{c} \Longleftrightarrow A<k$.

Proof : Lemma 1 implies that if $A \nless 0$ there is a constraint $\mathrm{c}$ for which $A^{c}$ has a nonnegative eigenvalue so that $A^{c} \nless 0$. Formula (3) implies that if $A<0$ then $A^{c}<0$ for all constraints $\mathrm{c}$. This proves the result for $k=0$. For $k \neq 0$ apply the result for $k=0$ to $A-k I_{n}$ and use $A^{c}-k I_{n-1}=Q^{T}\left(A-k I_{n}\right) Q$.

Lemma 3 (Calculation of $\operatorname{det} A^{c}$ when $A$ is singular) Suppose $A$ has eigenvalues $\lambda_{1}, \lambda_{2}, \ldots, \lambda_{n}$ with $\lambda_{1}=0$. The right and left eigenvectors corresponding to $\lambda_{1}$ are $\mathbf{v}$ and $\mathbf{w}$ respectively and $|\mathbf{v}|=|\mathbf{w}|=|\mathbf{c}|=1$. Then $\operatorname{det} A^{c}=\gamma(\mathbf{c}) \lambda_{2} \lambda_{3} \ldots \lambda_{n}$ where $\gamma(\mathbf{c})$ is given by $(4)$.

Proof: Write $W$ for the hyperplane through zero normal to $\mathbf{w}$. $W$ contains the range of $A$. We can write $A=A \pi_{v}$ where $\pi_{v}$ is the projection along $\mathrm{v}$ onto $W$ since $A(\mathbf{x}+k \mathbf{v})=A \mathbf{x}=A \pi_{v}(\mathbf{x}+k \mathbf{v})$ for $\mathbf{x} \in W$. The constrained vector field $A^{c}$ on $C$ is given by $A^{c}=\left.\pi_{c} A\right|_{C}=$ $\left.\pi_{c} A \pi_{v}\right|_{C}=\left.\pi_{c} \pi_{w} A \pi_{v}\right|_{C}=\left.\left.\left.\pi_{c}\right|_{W} \pi_{w} A\right|_{W} \pi_{v}\right|_{C}$ and

$$
\operatorname{det} A^{c}=\left.\left.\operatorname{det} \pi_{c}\right|_{W} \operatorname{det}\left(\left.\pi_{w} A\right|_{W}\right) \operatorname{det} \pi_{v}\right|_{C}
$$

where $\pi_{w}$ is the projection onto $W$ along $\mathbf{w}$. Now we calculate each of the factors of $\operatorname{det} A^{c}$ to obtain $\operatorname{det} A^{c}=$ $\gamma(\mathbf{c}) \lambda_{2} \lambda_{3} \ldots \lambda_{n}$.

First observe that $\operatorname{det}\left(\left.\pi_{w} A\right|_{W}\right)=\lambda_{2} \lambda_{3} \ldots \lambda_{n}$. (Generalized right eigenvectors $\left\{\mathbf{v}_{2}, \mathbf{v}_{3}, \ldots, \mathbf{v}_{\boldsymbol{n}}\right\}$ can form a basis for $W$ with respect to which $\left.A\right|_{W}$ has $\lambda_{2}, \lambda_{3}, \ldots, \lambda_{n}$ on the diagonal.) $\left.\pi_{c}\right|_{W}$ is the projection of $W$ onto $C$ along c. An $(n-1)$ volume $\Delta_{W}$ in $W$ projects under $\left.\pi_{c}\right|_{W}$ to an $(n-1)$ volume $\Delta_{W} \cos \angle W C$ in $C$ where $\angle W C$ is the angle between the hyperplanes $W$ and $C$. Thus $\left.\operatorname{det} \pi_{c}\right|_{W}=\cos \angle W C=\mathbf{w c}^{T}$. (Recall that the determinant of a map is its scaling of volumes.)

$\left.\pi_{v}\right|_{C}$ is the projection of $C$ onto $W$ along $\mathbf{v}$. Suppose an $(n-1)$ volume $\Delta_{C}$ in $C$ projects under $\left.\pi_{v}\right|_{C}$ to an $(n-1)$ volume $\Delta_{W}$ in $W$. Then both $\Delta_{C}$ and $\Delta_{W}$ project along $\mathbf{v}$ to the same $(n-1)$ volume $\Delta_{V}$ in the hyperplane $V$ normal to $\mathbf{v}$. Calculating as above we have $\Delta_{V}=\Delta_{C} \cos \angle C V=\Delta_{C} \mathrm{cv}$ and $\Delta_{V}=\Delta_{W} \cos \angle W V=$ $\Delta_{W} \mathbf{w v}$. Then $\Delta_{W}=\Delta_{C} \mathbf{c v} / \mathbf{w v}$ and $\left.\operatorname{det} \pi_{v}\right|_{C}=\mathbf{c v} / \mathbf{w v}$. (Note that $\mathbf{w v}$ is nonzero because $\mathbf{w v}=0$ implies that $\mathbf{w}\left\langle\mathbf{v}, \mathbf{v}_{2}, \mathbf{v}_{3}, \ldots, \mathbf{v}_{n}\right\rangle=0$ and $\mathbf{w}=0$, a contradiction.)

Lemma 4 (Stability of $A^{c}$ when $A$ is singular) Suppose $A$ has eigenvalues $\lambda_{1}, \lambda_{2}, \ldots, \lambda_{n}$ with $\lambda_{1}=0$ and $\lambda_{2}, \ldots, \lambda_{n}$ having real parts less than zero. The right and left eigenvectors corresponding to $\lambda_{1}$ are $\mathbf{v}$ and $\mathbf{w}$ respectively. Then $A^{c}$ is unstable if $\gamma(\mathbf{c})$ is negative and if $\gamma(\mathbf{c})$ is positive, $A^{c}$ has zero or an even number of eigenvalues with nonnegative real parts.

Proof: We choose $|\mathbf{c}|=1$ so that $\mathbf{c}$ varies over the $\mathrm{n}$ sphere $S^{n}$. However, c and $-\mathrm{c}$ represent the same hyperplane and may be identified so that $\mathbf{c}$ varies over projective space $\mathbb{P}^{n} . A^{c}$ and $\operatorname{det} A^{c}$ are continuous functions on $\mathbb{P}^{n}$. The value of $\operatorname{det} A^{c}$ partitions $\mathbb{P}^{n}$ into $D_{+}$, $D_{-}, D_{0}$ where

$$
\begin{aligned}
& D_{+}=\left\{\mathrm{c} \in \mathbf{P}^{n} \mid(-1)^{n-1} \operatorname{det} A^{c}>0\right\} \\
& D_{0}=\left\{\mathbf{c} \in \mathbf{P}^{n} \mid \operatorname{det} A^{c}=0\right\} \\
& D_{-}=\left\{\mathbf{c} \in \mathbf{P}^{n} \mid(-1)^{n-1} \operatorname{det} A^{c}<0\right\}
\end{aligned}
$$

Formula (4) shows that $D_{0}=\left\{\mathbf{c} \in \mathbb{P}^{n} \mid(\mathbf{c v})\left(\mathbf{w c}^{T}\right)=0\right\}$ so that $D_{0}$ corresponds in $S^{n}$ to the two great circles which are the intersection of $S^{n}$ with the two hyperplanes $\mathbf{c v}=0$ and $\mathbf{w c}^{T}=0 . D_{+}$and $D_{-}$are path connected open subsets of $\mathbb{P}^{n}$. It is clear from the value of $\operatorname{det} A^{c}$ that $A^{c}$ is unstable on $D_{-}$and singular on $D_{0}$.

Now we show that $A^{c}$ has zero or an even number of eigenvalues with nonnegative real parts on $D_{+}$. For the special case $\mathbf{c}=\mathbf{w} \in D_{+}$we have $C=\left\langle\mathbf{v}_{2}, \mathbf{v}_{3}, \ldots, \mathbf{v}_{n}\right\rangle$ and $A^{\mathrm{w}}$ has eigenvalues $\lambda_{2}, \lambda_{3}, \ldots, \lambda_{n}$ and is asymptotically stable. Now let $c_{1}$ be any point of $D_{+}$. Since $D_{+}$ is path connected, there is a path $\gamma:[0,1] \rightarrow D_{+}$with $\gamma(0)=\mathbf{w}$ and $\gamma(1)=\mathbf{c}_{1}$. Then $(-1)^{n-1} \operatorname{det} A^{\gamma(t)}>0$ for $t \in[0,1]$. In particular, $A^{\gamma(t)}$ does not become singular for $t \in[0,1]$ so that if eigenvalues of $A^{\gamma(t)}$ cross the imaginary axis they must cross in complex conjugate pairs. Hence $A^{c_{1}}$ has zero or an even number of eigenvalues with nonnegative real parts.

If $\gamma(\mathbf{c})<0$ then lemma 3 implies that $(-1)^{n-1} \operatorname{det} A^{c}$ $=(-1)^{n-1} \gamma(\mathbf{c}) \lambda_{2} \lambda_{3} \ldots \lambda_{n}<0$ and so $\mathrm{c} \in D_{-}$and $A^{c}$ is unstable. Similarly, if $\gamma(\mathbf{c})>0$ then $c \in D_{+}$and $A^{c}$ has zero or an even number of eigenvalues with nonnegative real parts.

Lemma 5 (Change in stability when a constraint is encountered) Suppose $A$ has eigenvalues $\lambda_{1}, \lambda_{2}, \ldots, \lambda_{n}$ with $\lambda_{1}$ real and having the maximum real part so that $\lambda_{1}>\operatorname{Re}\left\{\lambda_{i}\right\}, i=2,3, \ldots, n$. The right and left eigenvectors corresponding to $\lambda_{1}$ are $\mathbf{v}$ and $\mathbf{w}$ respectively. Then if $\gamma(\mathbf{c})$ is negative, $A^{c}$ has an eigenvalue with a real part greater than $\lambda_{\mathbf{1}}$. If $\gamma(\mathbf{c})$ is positive, then zero or an even number of eigenvalues of $A^{c}$ have real parts greater than or equal to $\lambda_{1}$. 
Proof: $A-\lambda_{1} I$ has a zero eigenvector with corresponding right and left eigenvectors $\mathbf{v}$ and $\mathbf{w}$ and all the other (generalized) eigenvalues $\lambda_{i}-\lambda_{1}, i=2,3, \ldots, n$ have negative real parts. Now apply Lemma 4 to $A-\lambda_{1} I$ and note that $A^{c}-\lambda_{1} I^{c}$ unstable implies that $A^{c}$ has an eigenvalue with a real part greater than $\lambda_{1}$ and $\left(A-\lambda_{1} I\right)^{c}=A^{c}-\lambda_{1} I^{c}$ having zero or an even number of eigenvalues with nonnegative real parts implies that zero or an even number of the eigenvalues of $A^{c}$ have real parts greater than or equal to $\lambda_{1}$.

\section{Application to power system stability}

We have an application to large scale electric power systems motivating this work. Voltage instabilities of power systems are of increasing concern and can lead to dynamic "voltage collapse" and blackout [1]. There is a possibility of improving system stability before voltage collapse by selectively applying the constraint of locking the tap-changing transformers used to regulate voltages in the network. The dynamics of tap-changing transformers are thought to contribute to the voltage collapse instability $[2,3,4]$. Vu and Liu [4] give an example in which locking a tap-changing transformer improves stability and avoids voltage collapse. The tap ratio $n$ is discrete, but its dynamics are often approximated $[2,3,4,5]$ by the differential equation

$$
\dot{n}=T_{s}^{-1}\left(V_{s}-V\right)
$$

where $T_{s}, V_{s}$ are constants and $V$ is the voltage magnitude to be controlled. Locking the tap ratio at its current value $n_{\text {lim }}$ (or $n$ reaching its limit $n_{\text {lim }}$ ) may be modelled by replacing equation (6) with $n=n_{\text {lim }}$. Suppose we are given a differential equation power system model including dynamics (6) of each of the tap-changing transformers and we wish to plan which tap-changing transformers to lock when the system is near voltage collapse. One approach is as follows: Assume the system is at the saddle node bifurcation of the operating equilibrium which is associated with voltage collapse $[1,6]$. Then the linearization $A$ of the system at the bifurcating equilibrium has a zero eigenvalue and the corresponding right and left eigenvectors $\mathbf{v}, \mathbf{w}$ can be computed. Locking the $i$ th tapchanging transformer is represented by imposing a constraint $\mathbf{c}_{\boldsymbol{i}}$. Lemma 4 applies and since the instability we wish to avoid is a saddle node bifurcation in which a single real eigenvalue of $A$ becomes positive, $\gamma\left(c_{i}\right)$ distinguishes the effect of $\mathbf{c}_{i}$ with respect to the instability. Indeed the tapchanging transformers which are most effective to lock are those with the largest $\gamma\left(\mathbf{c}_{i}\right)$. Tap-changing transformers with $\gamma\left(\mathbf{c}_{\boldsymbol{i}}\right)<0$ should not be locked as this would be destabilizing. In practice the computation of which tapchanging transformers to lock would be done using load flow equations rather than differential equation power system models. The computation can be adapted to the load flow equations although there is no space to explain this here. See [7] for a different approach to the control of tap ratios based on load power margin sensitivity.

\section{Conclusions}

Systems encountering constraints or limits and undergoing structural change are pervasive in engineering but are difficult to analyze. In this paper, we give a first analysis of the following case: We consider a nonlinear system at a quasistatically varying, stable equilibrium encountering a linear constraint on the system state and try to study the consequent change in system stability. We are able to make the following general observations, which depend on the Jacobian $A$ of the system at the equilibrium: If $A$ is negative definite then the constrained system is stable for all constraints. If $A$ is not nonnegative definite then there is a constraint which destabilizes the system. If $A$ is symmetric (e.g., the system is a gradient vector field on $\mathbf{R}^{n}$ ), then encountering the constraint cannot decrease the system stability. In the case of the eigenvalue of $A$ with maximum real part being real, there is a simple expression depending on the constraint and two eigenvectors of $A$ useful in describing some of the change in stability when the constraint is encountered. If the expression is negative, then the system is less stable when the constraint is encountered. If the expression is positive, this gives partial information about the change in stability when the constraint is encountered.

These results are a start to a stability theory of systems encountering constraints but are limited because they do not detect instability caused by complex pairs of eigenvalues crossing the imaginary axis. However the results are well suited to assessing the effect on stability of selectively locking tap-changer transformers when a power system is near a voltage collapse instability.

Support in part by NSF Research Initiation Grant ECS-9009079 is gratefully acknowledged.

[1] Proceedings:Bulk power system voltage phenomenavoltage stability and security, EPRI Report EL-6183, Potosi, Missouri, Jan. 1989.

[2] S. Abe, Y. Fukunaga, A. Isono, B. Kondo, "Power System Voltage Stability", IEEE Transations on PAS, vol. 101, No. 10, Oct. 1982, pp. 3830-3840.

[3] C.C. Liu, K.T. Vu, "Analysis of tap-changer dynamics and construction of voltage stability regions", IEEE Transations on CAS, vol. 36, No. 4, April 1989, pp. 575-590.

[4] K.T. Vu, C.C. Liu, "Dynamic mechanisms of voltage collapse", Systems and Control Letters, vol. 15, 1990, pp. 329-338.

[5] A.S. Debs, Modern Power Systems Control and Operation, Kluwer Academic, Boston, 1987.

[6] I. Dobson, H.-D. Chiang, "Towards a Theory of Voltage Collapse in Electric Power Systems," Systems and Control Letters, vol. 13, 1989, pp. 253-262.

[7] I. Dobson, L. Lu, Computing an optimum direction in control space to avoid saddle node bifurcation and voltage collapse in electric power systems. report ECE-91-2, ECE Dept., University of Wisconsin, Madison, WI 53706, Jan. 1991. 